\title{
Prise en charge de la dyspnée en fin de vie
}

\author{
Michael P. Slawnych MD PhD
}

— Citation : CMAJ 2020 May 19;192:E550. doi : 10.1503/cmaj.200488-f; diffusion hâtive le 21 avril 2020

Balados du CMAJ : Entrevue avec l'auteur (en anglais) accessible au : https://soundcloud.com/cmajpodcasts/200488-five. Voir la version anglaise de l'article ici : www.cmaj.ca/lookup/doi/10.1503/cmaj.200488

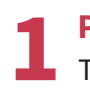

\section{Personne ne devrait mourir en détresse respiratoire}

Tous les médecins devraient être prêts à prendre en charge la dyspnée, surtout pendant la pandémie de maladie à coronavirus 201912. La dyspnée, soit l'expérience subjective d'une gêne respiratoire inconfortable, est habituellement bien traitée par des médicaments administrés par voie sous-cutanée; le papillon sous-cutané est une bonne option parce qu'il peut rester en place plusieurs jours, ce qui évite le besoin d'injections répétées².

2 Le traitement médicamenteux par opioïdes est la principale méthode de prise en charge de la dyspnée en fin de vie

On suggère que les médecins commencent le traitement avec les opoïdes ${ }^{3}$, qui ne modifient pas l'état respiratoire et n'accélèrent pas le décès lorsqu'ils sont utilisés de manière appropriée pour soulager les symptômes (p. ex., 0,5 mg d'hydromorphone en injection sous-cutanée toutes les 4 heures et $0,5 \mathrm{mg}$ par voie sous-cutanée toutes les 30 minutes, au besoin) $)^{4}$. La dose devrait être réévaluée fréquemment. Les médecins doivent être prêts à augmenter la dose rapidement si nécessaire. Les opioïdes administrés par voie sous-cutanée sont considérés comme 2 fois plus puissants que les opioïdes administrés par voie orale $(1,0 \mathrm{mg}$ d'hydromorphone administré par voie orale a à peu près le même effet que $0,5 \mathrm{mg}$ administré par voie sous-cutanée).

\section{Si la dyspnée persiste, une benzodiazépine peut être ajoutée}

Les benzodiazépines peuvent être utiles, particulièrement si le patient éprouve aussi de l'anxiété (p. ex., 0,5 mg de lorazépam administré par voie sous-cutanée toutes les 2 heures, au besoin [la dose peut être augmentée si nécessaire]). Pour une dyspnée réfractaire persistante, une sédation palliative pourrait être nécessaire. Un médecin en soins palliatifs devrait participer à ce traitement.

Les patients ont souvent des sécrétions gênantes en raison de

difficultés à avaler

Il n'y a habituellement pas lieu de faire une aspiration des sécrétions, intervention qui se révèle souvent inconfortable pour les patients. Les anticholinergiques sont les médicaments de choix pour réduire les sécrétions. Les médecins peuvent choisir entre la scopolamine et le glycopyrrolate selon les besoins en sédation $(0,4 \mathrm{mg}$ de scopolamine en injection sous-cutanée toutes les 4 heures, au besoin [sédation]; $0,4 \mathrm{mg}$ de glycopyrrolate en injection sous-cutanée toutes les 4 heures au besoin [absence de sédation]). Les bâtonnets de soins de bouche peuvent être utiles pour atténuer la sécheresse buccale.

\section{L'agitation associée à la dyspnée chez des patients peut bouleverser \\ 5 les proches}

Il est suggéré d'utiliser un antipsychotique pour réduire l'agitation. La méthotriméprazine est préférée en raison de ses propriétés sédatives (p. ex., 6,25 mg de méthotriméprazine en injection sous-cutanée toutes les 6 heures au besoin [la dose peut être augmentée si nécessaire]). Une benzodiazépine est parfois ajoutée, particulièrement à l'approche de la fin de la vie.

\section{Références}

1. Arya A, Buchman S, Gagnon B, et al. Pandemic palliative care: beyond ventilators and saving lives. CMAJ 2020;192:E400-4.

2. Goldstein NE, Morrison RS. Evidence-based practice of palliative medicine. Philadelphia: Elsevier Saunders; 2013.

3. Currow DC, Ward AM, Abernethy AP. Advances in the pharmacological management of breathlessness. Curr Opin Support Palliat Care 2009;3: 103-6.

4. Gallagher R. Killing the symptom without killing the patient. Can Fam Physician 2010;56:5446, e210-2.

Intérêts concurrents : Michael Slawnych signale avoir reçu des honoraires de conférencier en tant que membre d'une agence de conférenciers, Novartis.

Cet article a été révisé par des pairs.

Affiliations : Département de soins palliatifs et Institut de cardiologie Libin, Université de Calgary, Calgary, AB.

Correspondance : Michael Slawnych, michael.slawnych@ahs.ca 